\title{
Menger contre Walras
}

\author{
Sandye Gloria*
}

Il est courant de ne voir dans le rejet par Carl Menger de l'usage de l'outil mathématique qu'une spécificité anecdotique qui le différencie, sur la forme exclusivement, de l'analyse marginaliste de Léon Walras. Nous montrerons qu'en fin de compte le rejet des mathématiques (d'un certain type) par Menger est une décision cohérente parfaitement justifiée par la vision de la réalité économique que l'auteur cherche à rendre intelligible et par la méthode qu'il juge à cet effet appropriée. Certains outils aujourd'hui disponibles, les outils de la complexité, semblent bien adaptés à la pensée de cet auteur et permettent son renouvellement. Nous proposons les grandes lignes d'un programme néomengerien ancré dans l'économie de la complexité.

\section{MENGER VERSUS WALRAS}

It is common to see in the rejection by Carl Menger of the use of mathematics only an anecdotal specificity which differentiates the author, on the form exclusively, from the marginalist analysis of Léon Walras. We show that, ultimately, Menger's rejection of mathematics (of a certain type) is a coherent decision perfectly justified by the vision of economic reality which the author seeks to analyse and by the methods he judges most appropriated. Some tools available today, the tools of complexity, seem well adapted to the thinking of this author and allow his renewal. We propose the broad outlines of a Neo-Mengerian program rooted in complexity economics.

Mots clés : Menger, Walras, ontologie sociale, économie mathématique, économie de la complexité.

Keywords : Menger, Walras, social ontology, mathematical economics, complexity econommics.

Classification JEL : B13, B31, B41, B53, C65.

* Université Côte d'Azur, CNRS, GREDEG. Correspondance : Institut supérieur d'économie et de management, Campus Saint-Jean d'Angély 1, 24 avenue des Diables Bleus, 06357 Nice Cedex 4.Courriel : gloria@unice.fr

À la mémoire de Pierre Garrouste.

Je remercie Annie Cot pour m'avoir incitée à étudier cette question à l'occasion du Colloque « Contre Walras », Paris, 25-26 septembre 2015, organisé par le Centre d'économie de la Sorbonne où une première version de cet article a été présentée. Je remercie également Ludovic Ragni pour m'avoir orientée afin d'approfondir certains aspects de l'analyse walrassienne. Je remercie enfin les deux rapporteurs anonymes pour les judicieux conseils. Je demeure bien entendu seule responsable des éventuelles erreurs contenues dans cet article. 


\section{INTRODUCTION}

L'originalité de la tradition économique autrichienne s'est constituée, génération après génération, à travers la confrontation vis-à-vis de l'école walrassienne - puis néoclassique - à laquelle les auteurs autrichiens sont souvent assimilés. L'histoire de la constitution de la tradition économique autrichienne peut en effet être reconstruite comme un processus visant à se distinguer d'une école de pensée walrassienne englobante ${ }^{1}$.

Walras et ses disciples ont joué ce rôle à la fois de repoussoir et de point de repère pour une tradition autrichienne en quête d'originalité. En réalité, l'ambiguité et le paradoxe de la relation entre ces frères ennemis, si proches et si différents, tient au fait que si les termes utilisés sont souvent les mêmes - en particulier ceux de concurrence, équilibre, rationalité, subjectivisme - leur contenu théorique et les structures au sein desquelles ces concepts s'insèrent sont fort différents.

Il s'agit ici de montrer que, dès la première étape du processus historique de constitution des paradigmes autrichien et néoclassique, les dés sont jetés : Menger est de fait « contre Walras » et sa spécificité sera à l'origine d'une tradition dont la proximité avec la logique walrassienne de l'équilibre général n'est que superficielle ${ }^{2}$.

Cette contribution se positionne dans la continuité de Jaffé [1976]. Dans son célèbre article, Jaffé insiste sur les conséquences désastreuses pour la science économique d'une homogénéisation des travaux de Walras, Jevons et Menger. La révolution marginaliste est une construction des historiens de la pensée qui regroupent les trois auteurs sur la base d'une similitude de l'outil utilisé, à savoir l'utilité marginale. Jaffé ([1976], p. 512) reproche aux historiens de définir la science autour de l'outil (tooled knowledge) et de négliger les structures de connaissance (knowledge structures) auxquelles cet outil a donné lieu. Comme le rappelle justement Fontaine [1998], l'entreprise de dé-homogénéisation de Jaffé vise à corriger ce biais et à restaurer l'identité de chacun des auteurs en mettant en avant l'originalité de leurs édifices théoriques respectifs. Notre objectif est similaire puisqu'il s'agit en fin de compte de mettre au jour des potentialités théoriques que le processus d'homogénéisation a masquées. Jaffé ([1976], p. 521) conclut en identifiant chez Menger les prémisses de l'analyse stochastique et des propriétés informationnelles des systèmes économiques; notre entreprise de dé-homogénéisation a pour résultat de faire de Menger un précurseur de l'économie computationnelle, un théoricien propre à inspirer les développements modernes au sein du paradigme de la complexité en économie ${ }^{3}$.

1. Voir Gloria-Palermo [1996] pour une histoire détaillée du processus d'affirmation de l'originalité autrichienne.

2. En référence au colloque « Contre Walras ».

3. Il est important de préciser à ce niveau que le propos de cet article est limité à une confrontation de l'originalité mengerienne à la logique walrassienne de la théorie de l'équilibre général (TEG) et que sont laissés de côté les aspects économie sociale et économie appliquée de Walras. Cette réduction est acceptable pour notre propos dans la mesure où c'est bien au Walras de la TEG que Menger est généralement indistinctement ramené, que c'est bien au Walras des Éléments que Menger lui-même répond personnellement pour affirmer son originalité, et que c'est bien la TEG qui constituera par la suite le noyau dur s'il en est de l'économie mathématique moderne à laquelle les générations successives d'auteurs autrichiens seront à leur tour assimilées. Signalons toutefois qu'il n'est pas évident qu'une confrontation plus globale entre Menger et le Walras de l'économie sociale et appliquée aboutisse au même contraste, notamment en ce qui concerne les différences ontologiques qui seront soulignées au terme de l'analyse. 
Selon Walras, l'appartenance de Menger au mouvement révolutionnaire dont lui-même se veut l'instigateur ne fait aucun doute. Après lecture des Grundsätze de Menger, Walras écrit à l'auteur : « Nous aurions, vous et moi, le plus grand intérêt à nous mettre d'accord ; nous en serions beaucoup plus forts. Croyez, je vous prie, que je ferai mon possible pour y arriver » (lettre du 2 juillet 1883, dans Jaffé [1965], vol. I, p. 771). Par la suite encore : « Sachons nous comprendre et nous accorder et l'on verra bientôt l'avènement d'une école appelée à durer [...] et dont vous serez l'un des plus éminents promoteurs » (lettre du 4 avril 1887, dans Jaffé [1965], vol. II, p. 206).

La réponse de Menger aux lettres enthousiastes de Walras est cependant beaucoup plus retenue : "Une ressemblance existe bien entre nous. Il y a une analogie de concepts sur quelques points mais non sur les questions décisives » (lettre du 27 janvier 1887, dans Jaffé [1965], vol. II, p. 176, italique ajouté).

Cette réponse constituera le fil directeur de cet article : il s'agira dans la première section d'identifier cette analogie de concept, centrée essentiellement autour de l'utilité marginale. Quelques détails divergents sont pourtant déjà présents au terme de la confrontation. Ils représentent les symptômes des dissensions sur les questions décisives évoquées par Menger et qui concernent, d'une part, les dimensions temporelle et subjective des cadres théoriques respectifs (deuxième section) et, d'autre part, la méthodologie, avec l'adoption par Menger d'une démarche originale, l'approche causale-génétique (troisième section). Enfin (quatrième section), il s'agira de prendre encore plus de recul et d'identifier l'origine des dissensions au niveau le plus général, c'est-à-dire au niveau ontologique, et de montrer que la nature de la réalité économique qui sous-tend les analyses théoriques autrichiennes et marginalistes est distincte. Les outils mathématiques utilisés par Walras sont adaptés à l'objet des Éléments d'économie politique pure mais ne permettent pas de modéliser la réalité économique telle que l'appréhende Menger. Il existe toutefois une autre catégorie d'outils mathématiques (de type constructiviste) qui s'avère bien mieux adaptée à la nature de la réalité économique qu'envisagent les auteurs autrichiens. Ces outils permettent de renouveler le programme mengerien et d'inspirer la recherche contemporaine au sein de l'économie de la complexité.

\section{UNE ANALOGIE DE CONCEPTS ?}

Il est possible de comparer les concepts d'utilité marginale élaborés par Walras et Menger depuis différents angles de vue. Nous en voyons au moins quatre, chacun avec sa finalité propre.

L'angle le plus évident et le plus général consiste à insister sur la rupture avec la pensée classique qu'apporte l'introduction du concept d'utilité marginale, sans entrer dans le détail du contenu formel de ce que revêt ce concept pour chaque auteur. La révolution marginaliste est en effet précisément définie autour de la découverte simultanée et indépendante du principe de l'utilité marginale. Il s'agit de l'exemple typique en sciences économiques du phénomène de « découverte multiple » de Merton [1973] dans la mesure où Jevons, Menger et Walras élaborent un concept similaire alors même qu'ils sont issus de contextes universitaires distincts et qu'ils n'étaient que peu en contact les uns avec les autres. Cette découverte multiple résulterait essentiellement de l'insatisfaction partagée par les trois auteurs envers la théorie objective de la valeur qui définit la tradition 
économique classique. Il s'agit bien d'ailleurs pour Schumpeter ([1954], 1983, t. III, p. 220) d'une « révolution de la théorie de la valeur ». C'est précisément en réaction à cette pratique centrée sur l'outil et dont l'aboutissement est l'homogénéisation des auteurs sous le label de la révolution marginaliste que Jaffé écrit son fameux article.

Un autre angle de comparaison possible consiste à positionner les auteurs au sein du débat autour de la cardinalité ou de l'ordinalité de l'utilité marginale. Le niveau de généralité décroît, nous entrons progressivement dans le contenu formel de l'outil. Selon l'explication canonique, l'histoire du concept de l'utilité marginale est organisée en deux phases : une première phase qui va de 1870 à 1910 et qui regroupe Jevons, Menger et Walras autour d'une conception cardinale, et une deuxième phase qui va ensuite jusqu'à l'entre-deux-guerres avec Pareto et Hicks qui contribuent au développement d'une analyse ordinale.

Moscati [2013] dépasse la dichotomie et identifie une troisième catégorie, l'utilité classiquement mesurable. Moscati unifie rigoureusement Walras, Jevons et Menger autour de cette définition. Il s'agit de la catégorie la plus contraignante : dans la version ordinale, il est juste nécessaire que les individus sachent ordonner l'utilité des consommations alternatives ; dans la version cardinale, il faut en outre que les agents sachent ordonner les différences d'utilité ; dans la version classiquement mesurable, ou hypercardinaliste, il est de plus nécessaire d'identifier comme étalon de mesure une unité d'utilité afin de pouvoir déterminer les ratios d'utilité. Face aux critiques soulevées par la nécessité d'une mesure directe de l'utilité, Walras et Jevons développent des arguments relativement similaires. Les deux auteurs s'appuient sur l'exemple de la physique et de la mécanique où le concept de masse non directement mesurable est pourtant légitime ${ }^{4}$. Walras justifie sur la base de cette analogie sa démarche " as if » : "Supposons, pour un moment, que l'utilité soit directement mesurable, et nous nous trouverons en position de donner un compte rendu exact, mathématique de l'influence qu'elle exerce $[\ldots]$ sur les courbes de demande et donc sur les prix. Je vais, donc, faire l'hypothèse de l'existence d'un étalon de mesure de l'utilité intensive » (Walras [1874b], p. 16). La seule originalité de Menger ici est que, contrairement à Walras et Jevons, il ne cherche jamais à justifier la possibilité de mesurer l'utilité marginale, que ce soit pour éviter le problème ou parce qu'il n'en ressent pas la nécessité en raison du caractère non formalisé de sa représentation.

Le troisième angle de comparaison interroge la place du concept de l'utilité marginale au sein des édifices théoriques des deux penseurs. Il est pour Walras ([1874a], 1926, p. xvi) « le fondement de tout l'édifice ». L'un des objectifs récurrents de Walras est de faire de la rareté la cause de la valeur afin d'exprimer mathématiquement la théorie du juriste suisse Jean-Jacques Burlamaqui et de son père Auguste. Après plusieurs tentatives insatisfaisantes où Walras assimile fonction d'utilité et fonction de demande, l'auteur (Walras [1874b], p. 17) définit enfin la rareté comme utilité intensive, " l'intensité du dernier besoin satisfait par une quantité consommée ", et peut ainsi relier la rareté à la valeur des marchandises $^{5}$. Il est ici crucial de rappeler que l'idée d'un schéma analytique de marchés

4. Dans sa correspondance avec Poincarré, Walras se réfère aux Éléments de statique de Poinsot où la masse est définie comme le nombre de molécules qui composent un corps et, pourtant, « personne n'a jamais compté les molécules » (Jaffé [1965], vol. III, p. 161).

5. Voir Rebeyrol ([1999], p. 101-106) pour une présentation détaillée des méandres de la recherche walrassienne sur les liens entre rareté et valeur. Rebeyrol identifie les différentes pistes suivies par Walras avant d'aboutir à sa formulation définitive. 
concurrentiels interconnectés est développée par Walras bien avant. Dès 1860, dans son « Application des mathématiques à l'économie politique », l'auteur développe l'intuition de l'équilibre général mais sans relation entre l'utilité et la demande. La découverte du principe de l'utilité marginale est cruciale en ce qu'elle permet le traitement formel rigoureux du modèle d'équilibre général. C'est la dernière pièce du puzzle qu'il manquait à Walras afin de pouvoir présenter un modèle d'équilibre général cohérent. En ce sens, l'utilité est la cause logique de la valeur. Comme l'expliquent Bridel et Mornati ([2009], p. 882), pour Walras la structure de marché préexiste aux agents, l'auteur « descend » de sa théorie de l'équilibre général vers une théorie du comportement des agents qui la rend cohérente.

Le concept d'utilité marginale ne revêt pas le même rôle dans l'édifice mengerien. Elle est également cause de la valeur mais cause générative plutôt que logique. Menger emprunte sa conception de la causalité à Aristote et recherche derrière l'apparence des phénomènes leur cause essentielle, c'est-à-dire la circonstance qui permet au phénomène d'émerger. Ainsi lorsque Menger s'intéresse à l'échange, son objectif est là aussi de déterminer l'essence du phénomène, c'est-à-dire d'identifier la cause élémentaire à l'origine de son existence et le processus qui porte à son émergence. L'échange naît d'une évaluation compatible de la valeur des marchandises entre les agents et la valeur d'un bien est elle-même dérivée de l'intensité des besoins que la consommation de la dernière unité de ce bien permet de satisfaire pour chaque agent. Ainsi, le concept d'utilité marginale est nécessaire à Menger en ce qu'il permet de comprendre le processus qui amène des agents à échanger. Il est un maillon de la chaîne causale qui explique l'émergence du phénomène de la valeur dont le point de départ est le comportement de recherche de satisfaction des besoins des agents économiques.

Le dernier angle de comparaison que nous proposons d'approfondir maintenant consiste à entrer dans le détail proprement dit du contenu formel du concept. Dans sa fameuse table de l'intensité des besoins représentée ci-dessous (Menger [1871], 1950, p. 93), l'auteur classe en colonne de I à X les besoins subjectifs qu'un individu cherche à satisfaire par ordre d'importance décroissant. L'individu recherche parmi les biens disponibles ceux qui lui permettront au mieux de les satisfaire. Les biens ne sont donc qu'implicitement représentés. À chaque ligne correspond le supplément d'utilité que procure à l'individu la consommation d'une unité supplémentaire du bien associé au besoin de la colonne.

Figure 1. Table de l'intensité des besoins

Du besoin le plus urgent au moins urgent

\begin{tabular}{|c|c|c|c|c|c|c|c|c|c|c|}
\hline & & & & & US & tt a & jins & & & \\
\hline & 1 & II & III & IV & V & $\mathrm{VI}$ & VII & VIII & IX & $\mathrm{x}$ \\
\hline & 10 & 9 & 8 & 7 & 6 & 5 & 4 & 3 & 2 & 1 \\
\hline & 9 & 8 & 7 & 6 & 5 & 4 & 3 & 2 & 1 & 0 \\
\hline Degré de & 8 & 7 & 6 & 5 & 4 & 3 & 2 & 1 & 0 & \\
\hline satisfaction & 7 & 6 & 5 & 4 & 3 & 2 & 1 & 0 & & \\
\hline pour chaque unité & 6 & 5 & 4 & 3 & 2 & 1 & 0 & & & \\
\hline consommée & 5 & 4 & 3 & 2 & 1 & 0 & & & & \\
\hline & 4 & 3 & 2 & 1 & 0 & & & & & \\
\hline & 3 & 2 & 1 & 0 & & & & & & \\
\hline & 2 & 1 & 0 & & & & & & & \\
\hline & 1 & 0 & & & & & & & & \\
\hline
\end{tabular}


Cette table permet cependant de mettre en lumière deux nuances qui distinguent Menger des représentations walrassienne et jevonienne.

Il est intéressant de noter que la satisfaction d'un besoin est obtenue à partir de la consommation d'unités concrètes de biens, Menger se référant à « des quantités économiquement admissibles » et ne passant jamais du discret au continu, ce qui lui aurait permis de construire de véritables courbes d'utilité marginale décroissante.

Menger ne relie pas directement l'utilité d'un bien aux quantités consommées ; la table relie l'intensité de la satisfaction à la marge au besoin satisfait. Menger insiste ainsi sur l'idée que la cause essentielle de la valeur des biens ne réside pas dans la quantité consommée mais est à rechercher au niveau de la nature du besoin même que cette quantité consommée permet de satisfaire. Cette distinction qui peut sembler a priori anecdotique est en réalité déterminante aux yeux d'un auteur dont l'objectif est de cerner les causes essentielles des phénomènes économiques au-delà de leurs manifestations symptomatiques. Dans cette perspective, la consommation d'une certaine quantité d'un bien n'est que la manifestation concrète, on pourrait dire accidentelle, du moyen par lequel un besoin est comblé et c'est la satisfaction de ce besoin qui est à l'origine de la valeur du bien.

La représentation que donne Menger du principe de l'utilité marginale diffère ainsi sur quelques détails qui sont en réalité révélateurs de l'écart avec la théorie de Walras. Le travail de Karl Menger [1973], fils mathématicien du fondateur autrichien, permet d'approfondir cette idée. L'auteur fournit une formulation mathématique relativement fidèle de la définition autrichienne du principe de l'utilité marginale ${ }^{6}$ :

Soient $x_{1}, x_{2}$ et $h$ des quantités différentes d'un même bien, telles que $x_{1}<x_{2}$ et $f$, la fonction de satisfaction de l'agent concerné, alors,

$$
\left|f\left(x_{1}+h\right)-f\left(x_{1}\right)\right| \geq\left|f\left(x_{2}+h\right)-f\left(x_{2}\right)\right|,
$$

et pour toute quantité $x$ du bien,

$$
f(x)-f(x-h) \geq f(x+h)-f(x),
$$

c'est-à-dire :

« [(1) :] Si deux quantités d'un bien sont toutes deux augmentées ou toutes deux diminuées du même montant, alors l'utilité de la quantité la plus importante varie moins (ou en tout cas pas plus) que celle de la quantité plus faible. [(2) :] Pour n'importe quelle quantité d'un bien, l'augmentation de l'utilité, consécutive à l'augmentation de cette quantité, est moindre (ou en tout cas pas plus grande) que la perte d'utilité consécutive à la diminution de la même quantité » (Menger [1973], p. 43).

La formalisation marginaliste consiste à définir l'utilité $u$ comme une fonction croissante à taux décroissant des quantités consommées. Cette définition implique la continuité et la différentiabilité des fonctions d'utilités, hypothèses non invoquées par la version autrichienne fournie par Menger fils :

$$
u=f(x), \text { telle que } f^{\prime}(x) \geq 0 \text { et } f^{\prime \prime}(x) \leq 0 .
$$

6. Relativement fidèle : le bémol concerne le fait que Karl Menger présuppose des fonctions qui relient directement l'utilité à la quantité consommée de bien. 
Ainsi, la formalisation de la théorie autrichienne est bien distincte de la représentation marginaliste ; elle sous-tend une vision de l'agent économique raisonnant sur des préférences discrètes et non généralisables en une fonction d'utilité continue. Cette distinction est déjà notée par Jaffé ([1976], p. 521) : « Loin d'être un "calculateur éclairé", il est une créature maladroite, errante, mal informée, tourmentée par l'incertitude... congénitalement incapable de former des décisions définitives et calibrées afin de poursuivre ses satisfactions. L'échelle de Menger, illustrant la décroissance dans l'importance des satisfactions, est représentée à travers des entiers discrets. Dans le schéma mengerien de pensée, il n'y a pas de place pour des dérivées premières positives par rapport aux quantités et des dérivées secondes négatives : rien n'est différentiable. »

Il s'agit maintenant d'approfondir les conséquences de cette distinction. L'environnement dans lequel évolue l'agent économique mengerien n'est pas figé mais en continuelle mutation. Il n'est pas possible de maximiser des fonctions d'utilité car de telles fonctions n'existent simplement pas. La prise en compte de cette spécificité ontologique entraîne des différences de fond importantes entre les deux auteurs sur la façon dont est conceptualisée la rationalité individuelle.

\section{DIMENSION TEMPORELLE ET SUBJECTIVISME DYNAMIQUE}

Ainsi, bien loin d'être un détail, la nuance formelle révélée par Karl Menger est la manifestation d'une profonde dissension sur une question décisive. Elle concerne précisément la dimension subjective de l'agent économique. A priori le subjectivisme est une caractéristique commune aux approches de Menger et de Walras en rupture avec la théorie objective de la valeur classique. Mais en réalité Menger pose les bases de ce que O'Driscoll et Rizzo [1985] définiront plus tard comme le subjectivisme dynamique, bannière de ralliement des Autrichiens modernes et qui s'oppose au subjectivisme statique sur lequel s'appuie Walras pour caractériser son agent économique.

Au cœur du clivage se trouve la façon dont les deux auteurs considèrent la dimension temporelle au sein de leur analyse. Il existe certes des éléments d'analyse dynamique chez Walras, et aussi serait-il caricatural d'opposer sans précaution préalable une analyse mengerienne dynamique à une approche walrassienne statique et atemporelle. Cela étant dit, il ressortira de la confrontation que les éléments dynamiques sont l'exception au sein d'un schéma walrassien où fondamentalement la dimension temporelle est appréhendée selon une approche logique qui contraste irrémédiablement avec une analyse mengerienne dynamique dans son essence.

Le concept de concurrence dans l'édifice walrassien revêt diverses significations : dans le cadre de l'économie politique pure, nous connaissons la concurrence comme un processus virtuel de tâtonnement où les échanges sont « suspendus » (Walras [1874a], 1889, p. 72) tant que les prix proposés ne correspondent pas aux prix d'équilibre et où la production se fait par l'entremise de bons tant que là aussi l'équilibre n'est pas calculé. C'est cette représentation atemporelle où la concurrence est un algorithme permettant logiquement de déterminer l'une des façons de rejoindre la solution d'équilibre qui a par la suite formé le cœur du programme de recherche néowalrassien. 
Mais si l'on revient à la démarche de Walras, au rapport qu'il pose entre théorie pure et théorie appliquée, il est possible de mettre en avant une autre conceptualisation de la concurrence qui vient nuancer l'interprétation statique précédente. Walras ([1874a], 1900, p. 30) part de la concurrence comme fait réel ou type réel et en abstrait un type idéal, objet de sa formalisation et qui a le rôle de norme guidant par la suite les interventions de politique économique : « [...] l'Économie Politique Pure doit emprunter à l'expérience des types d'échange, d'offre, ou de demande, de marchés, de capitaux, de revenus, de services producteurs, de produits. De ces types réels, elle doit abstraire, par définition, des types idéaux, et raisonner sur ces derniers, pour ne revenir à la réalité que la science une fois faite et en vue des applications. »

Le tâtonnement virtuel s'applique à un type idéal de marché particulier, le « marché une fois pour toutes » où les données fondamentales de l'économie sont invariantes et la solution est l'équilibre général ; mais dans son souci de revenir au réel Walras passe au type idéal du " marché temporaire » où les fondamentaux changent d'une période à l'autre, notamment les dotations initiales de chaque période qui sont héritées du résultat de l'interaction marchande de la période précédente (les stocks de services producteurs et de biens capitaux neufs). Le mécanisme concurrentiel ici en œuvre à l'intérieur de la période ne diffère cependant pas de celui du tâtonnement virtuel atemporel précédemment mobilisé.

C'est avec la définition d'un troisième type idéal de marché que Walras (1874a, 1988, p. 579-580) rompt avec la virtualité du mécanisme concurrentiel : « Enfin pour nous rapprocher de plus en plus de la réalité des choses, nous devons encore passer de l'hypothèse d'un marché périodique annuel à celle du marché permanent, c'est-à-dire de l'état statique à l'état dynamique. » C'est donc quand il introduit le « marché permanent » dans la septième et avant-dernière section des Éléments que, comme l'expliquent parfaitement Arena et Ragni [1994], le mécanisme de concurrence devient dynamique. Les fondamentaux varient constamment et la solution du modèle donnée par Walras ([1874a], 1988, p. 579-580) n'est plus mathématique mais intuitive ${ }^{7}:$ «Tel est le marché permanent, tendant toujours à l'équilibre sans y parvenir jamais pour la raison qu'il ne s'y achemine que par tâtonnement et qu'avant même que ces tâtonnements ne soient achevés, ils sont à recommencer sur de nouveaux faits, toutes les données du problème telles que les quantités possédées, utilités des produits et des services, coefficients de fabrication, excédent du revenu sur la consommation, exigence des fonds de roulement, etc., ayant changé. »

Interrogeons-nous encore sur la place respective de la concurrence comme mécanisme virtuel et de la concurrence comme mécanisme réel et dynamique dans la globalité de l'œuvre walrassienne. Si effectivement le but de la science est de se rapprocher du réel alors le modèle du marché permanent serait l'aboutissement du projet walrassien. Mais c'est exactement le contraire qui motive le projet de l'auteur, comme il l'explicite à l'occasion d'une critique à son successeur à Lausanne, Wilfredo Pareto : «M. P. croit que le but de la Science est de se rapprocher de plus en plus de la réalité par des approximations successives. Et moi je crois que le but final de la Science est de rapprocher la réalité d'un certain idéal ; c'est pourquoi je formule cet idéal » (Walras [2000], p. 567). Certes le

7. C'est essentiellement sur cette base que Walker [1987] fait du modèle walrassien originel un modèle de déséquilibre où les entrepreneurs subissent des pertes effectives et réalisent des profits qui influent de par leurs décisions d'entrée et de sortie de branche sur les quantités et tendent à ajuster les prix. 
marché permanent est plus réaliste mais son idéal est le marché une fois pour toutes $^{8}$. Le marché une fois pour toutes (et le marché périodique) est un type idéal dont l'intérêt scientifique est celui de procurer une norme « comme en mécanique pure on suppose d'abord des machines sans frottement » (Walras [1874a], 1900, p. 45), norme qui permet le passage à l'économie appliquée et à l'intervention de l'État pour les situations différant substantiellement de cet idéal. Dans les deux cas du marché une fois pour toutes et du marché temporaire justifiés par l'analogie à la mécanique sans frottement, l'analyse est indubitablement statique, avec, pour reprendre Ménard [1979], une incapacité congénitale à introduire le temps.

L'étendue de l'écart entre Menger et Walras est de ce point de vue parfaitement synthétisée par Streissler ([1972], p. 440) lorsqu'il remarque que « le tâtonnement walrassien prend une minute, le tâtonnement mengerien prend un siècle ». Au temps logique, newtonien, de Walras, Menger oppose une conception bergsonienne du temps, c'est-à-dire que le temps passe selon la perception subjective des agents ${ }^{9}$; l'écoulement du temps est en soi source de changement, de nouveauté, venant modifier sans cesse les informations sur lesquelles les agents se basent pour prendre leurs décisions. La concurrence est véritablement processus ; en tant qu'interaction réelle et non virtuelle elle est l'occasion pour les individus de corriger et améliorer les informations à partir desquelles ils élaborent leurs schémas décisionnels fins-moyens. La principale cause de progrès économique réside d'ailleurs pour Menger dans l'amélioration des connaissances. La recherche d'information est le moteur du progrès : si au gré de l'interaction économique, les individus acquièrent de meilleures connaissances dans les relations causales qui dirigent l'activité économique, l'incertitude et les risques d'erreur d'évaluation diminuent, permettant de mettre en place des processus productifs et d'échange plus précis et mieux adaptés aux besoins. «Une compréhension plus approfondie des connexions causales entre les biens et le bien-être humain, d'une part, et un contrôle plus précis des conditions dont dépend ce bien-être d'autre part, ont amené la condition humaine d'un état de barbarie et de profonde misère vers le présent état de civilisation et de bien-être [...]. Rien n'est plus évident que le fait que le degré de progrès économique de la condition humaine, sera toujours, pour les périodes futures, fonction du degré de progrès dans les connaissances humaines » (Menger [1871], 1950, p. 74).

La prise de décision ne consiste pas à choisir la solution optimale au sein d'un ensemble fermé d'alternatives identifiées mais, à travers l'acquisition de nouvelles connaissances permises par le processus concurrentiel, à constamment reformuler les plans fins-moyens qui fondent les choix individuels. « Dès lors que nous acceptons l'idée que le temps s'écoule, nous devons permettre à la connaissance de se modifier, et la connaissance ne peut être appréhendée comme une fonction de quelqu'autre argument que ce soit » (Lachmann [1976], p. 127-128). L'Homo agens autrichien se distingue ainsi de l'Homo æeconomicus walrassien et ne se réduit pas à un calculateur-maximisateur réagissant de façon automatique à son environnement. L'on comprend en effet qu'il serait dès lors incohérent de capturer la subjectivité (dynamique) des agents au sein de relations fonctionnelles stables dans le temps puisque dans ce cadre les échanges ne sont pas suspendus

8. L'interprétation donnée par Bridel et Mornati [2009] des relations entre Pareto et Walras est basée sur la primauté entre réalisme ou abstraction que privilégient les deux auteurs ; Pareto craint que le primat de l'instrument mathématique sur le réalisme ne fasse de l'économie pure walrassienne une branche de la métaphysique.

9. Voir Rizzo [1994] pour cette interprétation bergsonienne du temps chez Menger. 
et le temps qui passe modifie sans cesse les données sur lesquelles se basent les agents pour formuler leurs plans fins-moyens.

La théorie de la production qu'offre Menger illustre parfaitement le rôle spécifique que joue le temps dans son analyse. Les biens de consommation constituent le moyen le plus direct pour satisfaire les besoins. Menger appelle ces biens économiques les biens « de premier rang » pour marquer leur proximité avec l'objectif d'accomplissement des besoins. Les biens « de second rang » sont les biens qui sont transformés en une seule étape de production en biens de premier rang. Plus généralement, les biens « de rang supérieur» font référence aux moyens de production : il s'agit des biens qui concourent de façon plus ou moins indirecte à la satisfaction des besoins à travers le processus de production. Le rang d'un bien n'est pas une caractéristique intrinsèque de l'objet mais dépend de la nature du processus productif au sein duquel il s'insère, ce processus pouvant être modifié au gré des connaissances techniques qu'acquièrent les agents économiques. Un rang élevé signifie que le bien répond de façon plus indirecte et détournée à la satisfaction des besoins qu'un bien de rang inférieur au sein du même processus productif.

La classification des biens selon leur rang permet à Menger de définir la production comme « un processus causal d'interconnexion entre les biens de différents rangs ». Il s'agit d'un processus séquentiel qui s'inscrit dans le temps. «L'idée de causalité, explique Menger ([1871], 1950, p. 67-68), [...] est inséparable de l'idée de temps [...]. Une procédure de changement implique un début et un devenant et ceux-ci ne sont concevables que comme des processus dans le temps. Aussi courtes que soient les périodes de temps entre les différentes phases du procès [productif] [...], leur effacement complet demeure cependant inconcevable. »

L'introduction de la dimension temporelle amène donc irrémédiablement le facteur incertitude dans l'analyse puisqu'il existe un délai incompressible entre le moment où sont réunis les biens de plus hauts rangs et l'obtention du bien de premier rang correspondant. En début de processus, il y a incertitude quant à la quantité et la qualité du bien qui sera en fin de compte disponible pour satisfaire des besoins qui eux-mêmes sont en constante évolution. Ce type d'incertitude est incontournable selon Menger. Le fait que la production prenne du temps impose ainsi aux agents mettant en œuvre l'activité économique de procéder à des anticipations quant aux résultats qu'ils envisagent. Plus précisément, l'activité économique consiste pour l'agent à planifier les biens qui sont nécessaires directement ou indirectement à la satisfaction de ses besoins. La production devient pour les individus « une tentative de combler à l'avance leurs exigences » (Menger, ibid., p. 79). L'entrepreneur doit anticiper les besoins futurs de ses clients avant de mettre en œuvre le processus de production. Le passage du temps est en soi une source de changement et de nouveauté qui vient modifier les informations sur lesquelles se fondent les acteurs pour formuler leurs anticipations et prendre leurs décisions. L'activité productive est en ce sens analysée comme une activité subjective.

\section{MÉTHODE FONCTIONNELLE VERSUS MÉTHODE CAUSALE-GÉNÉTIQUE}

Au-delà de cette dissension conceptuelle autour de la dimension temporelle et de ses conséquences sur la nature du subjectivisme et la caractérisation de l'agent économique, revenons aux sources : quel est l'objet de la discipline ? Quelles sont 
les questions que doit se poser l'économiste et par quelles méthodes devrait-il s'employer à y répondre ? C'est en amont sur ces questions essentielles que se construit déjà le fossé qui sépare Menger de ses compagnons marginalistes.

Walras et Jevons définissent clairement les objectifs analytiques qu'ils poursuivent, Walras cherchant à élaborer avec rigueur une théorie de la détermination des prix relatifs d'équilibre en situation de concurrence pure et parfaite, et Jevons souhaitant définir la science économique comme un exercice de maximisation de la satisfaction des agents. L'objectif de Menger ne relève pas quant à lui de la pure logique de l'allocation. Son ambition est autre et relève avant tout de la méthode. Son objectif est de faire de la science économique une science théorique. Rappelons-nous qu'alors l'école historiciste allemande est dominante, le travail de l'économiste est inductif et consiste à identifier des régularités empiriques issues de la collecte de données et de l'analyse de situations historiques spécifiques. Pour Menger, la science économique est au contraire un ensemble de lois théoriques universelles. Pour établir ces lois, les phénomènes économiques compliqués doivent être décomposés en leurs éléments les plus simples, une décomposition logique en termes de causalité (Menger [1871], 1950, p. 46-47). Menger identifie l'élément explicatif le plus simple à la base de toutes les chaînes de raisonnement comme étant le principe de l'accomplissement (Bedürfnissbefriedigung), à savoir le principe selon lequel les individus sont guidés par la recherche de la satisfaction de leurs besoins. Il s'agit là de l'élément causal essentiel à la source de la compréhension de tous les phénomènes économiques complexes, comme les prix monétaires, la production ou la monnaie.

Dans son travail de 1888, Untersuchungen über die Methode (Investigations sur la méthode), le fondateur autrichien approfondit les principes méthodologiques qui doivent guider toute science théorique et en particulier l'économie : l'essentialisme et l'universalisme. L'approche scientifique, dont l'objet est d'acquérir une connaissance générale des phénomènes, consiste en la recherche systématique des causes ultimes - essentielles - à l'origine de ces phénomènes, à travers l'établissement de lois générales ne souffrant d'aucune exception. Comprendre un phénomène signifie donc identifier le processus causal à l'origine de sa genèse, en démarrant de la cause la plus élémentaire - le principe de l'accomplissement jusqu'à la manifestation la plus articulée du phénomène analysé. Plus précisément, Menger ([1883], 1963, p. 146) se pose la question suivante : « Comment se fait-il que les institutions qui servent le bien-être collectif et qui contribuent de façon très significative à son développement, émergent sans qu'une volonté commune soit dirigée vers leur établissement?» Une page plus loin, il précise enfin qu'il s'agit bien là de la question fondamentale que doit tenter de résoudre le théoricien des sciences sociales : « La solution aux plus importants problèmes des sciences sociales théoriques en général et de la théorie économique en particulier est ainsi directement reliée à la question de la compréhension théorique de l'origine et du changement des structures sociales “organiquement" créées » (ibid., p. 147). L'analyse d'une institution économique comme celle de tout autre phénomène économique consiste pour Menger à analyser le processus qui porte à son émergence en partant du principe de l'accomplissement.

Menger distingue deux types d'institutions : les institutions « organiques » et les institutions « pragmatiques ». La première catégorie concerne les phénomènes sociaux d'origine spontanée alors que la seconde catégorie regarde les organisations sociales qui résultent d'une décision centralisée imposée par voie légale ou d'un accord explicite entre les individus. Comprendre le processus d'émergence 
des institutions pragmatiques n'est donc par définition pas une question pertinente et tout l'enjeu réside dans la maîtrise du processus qui conduit à l'établissement des institutions organiques. Telle est pour Menger la question fondamentale qui doit canaliser les efforts d'investigation des théoriciens de la science économique. L'auteur se concentre ainsi sur l'analyse de l'essence des phénomènes sociaux organiques qu'il définit plus précisément comme des institutions qui «s'avèrent ne pas être le résultat d'une intention consciemment orientée vers ce but, i.e., le résultat d'un accord des membres de la société ou d'une législation positive. Elles se présentent à nous plutôt comme des produits naturels (dans un certain sens), comme les résultats inattendus du développement historique » (ibid., p. 131, en italique dans l'original).

Dans ce domaine l'illustration la plus connue que développe Menger est indubitablement son analyse de l'émergence de la monnaie. Aucune analyse comparable ne se retrouve chez Walras qui se concentre plutôt sur les caractéristiques optimales d'un système monétaire destiné à assurer la justice dans le fonctionnement de l'économie. En d'autres termes, l'objet d'investigation est différent : Menger répond à la question de l'origine de la monnaie ; il procède en identifiant le processus d'émergence de cette institution organique en des termes évolutionnistes témoignant de la modernité d'une approche qui continue aujourd'hui d'inspirer de nombreux théoriciens monétaires (voir Latzer et Schmitz [2002]) ; Walras, quant à lui, part du point d'arrivée et adopte le point de vue fonctionnel consistant à identifier le système monétaire optimal qui permet la justice sociale, c'est-à-dire l'unicité et la stabilité des prix ${ }^{10}$.

Il est possible de reconstituer à partir des écrits de Menger l'analyse d'une autre catégorie d'institution organique, le marché. L'auteur développe cette analyse dans le but de préciser la définition du concept de marchandise, le fait qu'il décrive l'émergence de la division du travail et du marché comme institution organique n'étant qu'un produit joint ; le parallèle avec le processus d'émergence de la monnaie nous semble cependant évident. Il s'agit d'après notre interprétation d'une illustration de ces nombreuses institutions sociales organiques auxquelles il se réfère par la suite dans ses Investigations : «On aurait pu exposer de la même manière [que l'analyse de l'origine de la monnaie] ce qu'il en ait des autres institutions sociales [...]. L'organisation de la circulation des marchandises sur des marchés qui reviennent périodiquement dans des lieux déterminés, l'organisation des métiers et de la division du travail, les usages du commerce, etc., ce sont là les institutions les plus éclatantes qui servent éminemment les intérêts du bien commun et dont l'origine semble, à première vue, devoir se rapporter nécessairement à une convention ou à la contrainte exercée par l'État, mais qui ne résultent pourtant pas à l'origine d'un accord, d'un contrat, d'une loi ou d'une prise en compte particulière de l'intérêt public de la part des individus singuliers, et qui procèdent au contraire d'aspirations servant des intérêts individuels » (Menger [1883], 2011, p. 301-302).

10. Walras propose comme solution un système de monnaie or avec billon d'argent régulateur ([1886], 1992, p. 108-109) qui permettrait selon lui d'éviter « un trouble général de l'équilibre économique » (ibid., p. 97) qui ne manquerait de se produire si la rareté relative et donc la valeur de la monnaie venait à se modifier. Voir en particulier sur ce thème Jacoud [1994]. Pour une confrontation détaillée complète de Walras et Menger sur la monnaie (son origine mais aussi ses fonctions et sa mesure), voir Arena et Gloria-Palermo [2008] et Álvarez et Bignon [2013]. 
Outre le fait que cette analyse est beaucoup moins connue, elle est intéressante pour deux autres raisons. Elle permet d'abord de confirmer l'importance du thème des institutions au cœur de la pensée mengerienne. Elle permet également de vérifier la cohérence de la démarche de l'auteur puisqu'en en effet l'analyse du marché est abordée selon la même logique : il s'agit de comprendre cette institution à travers l'identification du processus qui aboutit à son émergence comme résultat inattendu de l'interaction individuelle. L'auteur (Menger [1871], 1950, p. 236-239) analyse comment l'économie évolue du ménage isolé au marché organisé. L'économie du ménage isolé correspond à un système autarcique où aucun échange n'a lieu et la production se fait en vase clos; les biens sont distribués par une autorité familiale centrale selon une règle a priori de division du travail. Pour pallier les limites de ce système qui ne permet pas de couvrir la variété des besoins individuels, certains individus ont l'idée d'offrir leurs services d'artisans directement aux consommateurs. Lors de cette étape, les consommateurs fournissent les matières premières et offrent une compensation aux artisans qui apportent leur dextérité. Une nouvelle étape est franchie lorsque les artisans prennent l'initiative de fournir eux-mêmes les matières premières. L'économie se transforme ainsi progressivement en un nouveau système de production sans marché, l'économie de production sur ordre. Ce système présente encore de sérieux désavantages : les consommateurs doivent patienter avant d'accéder à un produit dont ils ne peuvent être sûrs à l'avance de la qualité et les artisans ne peuvent maitriser le rythme des commandes. Menger explique alors comment une nouvelle catégorie d'individus ingénieux, les intermédiaires, trouvent une façon d'améliorer l'organisation de l'économie tout en en tirant des bénéfices propres. Les intermédiaires commandent des biens de premier ordre aux artisans mais, contrairement aux consommateurs, l'objectif n'est pas la consommation et la satisfaction de leurs besoins ; ils conservent ces marchandises pour pouvoir les vendre aux consommateurs qui ont ainsi accès directement et sans délai aux biens de premier ordre qu'ils désirent. La division du travail se met spontanément en place, les biens produits ainsi à l'avance acquièrent le statut de marchandise économique. La statut de marchandise n'est pas dû à une quelconque qualité intrinsèque de l'objet mais découle de la relation spécifique et transitoire que ce bien entretient avec la personne qui l'a commandé.

Dans les deux cas de l'analyse de l'émergence de la monnaie et du marché organisé, les institutions organiques résultent de l'interaction de deux types de rationalité sans intervention centralisée. D'un côté, des individus ingénieux mettent en place de nouvelles façons de procéder qui répondent à la fois mieux à leurs propres besoins mais contribuent également à améliorer le fonctionnement global de l'économie ; de l'autre côté, des agents plus passifs réalisent progressivement les avantages qu'ils pourraient retirer de l'adoption des innovations introduites par la première catégorie d'agents. Leur décision d'imiter les innovateurs contribue à la diffusion d'une nouvelle norme économique.

Lorsque Menger adopte cette démarche à la compréhension du phénomène des prix monétaires, l'écart avec le point de vue walrassien devient frappant. Il s'agit pour l'auteur des Grundsätze d'identifier le processus qui, partant de la cause essentielle du phénomène - l'individu et le principe de l'accomplissement -, retrace le cheminement par lequel les prix s'établissent. Le plus important pour Menger n'est donc pas d'élaborer une théorie de la détermination des prix d'équilibre, objet central de la réflexion walrassienne s'il en est, mais plutôt de comprendre l'essence du phénomène de l'échange dont l'aboutissement visible, 
le symptôme, est la fixation d'un prix monétaire. La précision formelle n'est pas son souci principal, il cherche en fait à maîtriser la chaîne causale décrivant le processus de l'échange, qui part de l'individu et aboutit aux prix relatifs.

Dans quelle mesure deux individus sont-ils incités à échanger les biens qu'ils possèdent? Il n'y a échange que si les deux agents y trouvent chacun un avantage, étant donné l'évaluation subjective qu'ils font de leurs besoins et de la capacité des biens objets de l'échange à les satisfaire. Plus précisément, il y aura possibilité d'échange si un individu possède une quantité d'un bien de moindre valeur à ses yeux que la quantité d'un autre bien possédée par un autre individu qui présente des évaluations opposées. Le niveau des prix relatifs entre les deux biens se situe à l'intérieur des limites définies par la différence entre les évaluations respectives des deux agents ${ }^{11}$. Le niveau final du prix relatif résultera du rapport de forces entre les deux agents lors de la négociation; Menger évoque la possibilité d'intervention de causes extra-économiques pouvant influer sur ce rapport de forces de sorte que la théorie économique ne peut que préciser les limites de l'intervalle à l'intérieur duquel le prix se fixera mais non son niveau définitif. Un tel résultat théorique, somme toute imprécis et peu satisfaisant pour les marginalistes, est parfaitement légitime pour le propos mengerien de recherche de l'essence des phénomènes. Menger ([1871], 1950, p. 194) n'hésite pas à expliquer que la « formation des prix demeure indéterminée sur une certaine plage, sans que cela ne fasse perdre à l'échange son caractère économique. L'étendue de cette plage dépend de la nature et des conditions particulières de l'échange ». Nous sommes ici bien loin du monde parfait et dénué d'incertitude que décrit Walras. Le système mengerien n'est pas fermé, tous les déterminants ne sont pas maîtrisés et la théorie ne peut que définir un intervalle de prix relatifs.

Partant précisément de la confrontation des théories des prix de Menger et Walras, Hans Mayer ([1932], 1994, p. 57), disciple du fondateur autrichien, distingue en ces termes les deux approches : du côté autrichien, les prix sont expliqués à travers une démarche dite causale-génétique qui, « en expliquant la formation des prix, a pour but de fournir une compréhension des corrélations de prix à travers la connaissance des lois de leur genèse ", et les approches fonctionnelles qui, « en déterminant précisément les conditions de l'équilibre, ont pour but de décrire la relation de correspondance entre des prix déjà existante en situation d'équilibre ». Ainsi la théorie des prix mengerienne vise à identifier le processus causal qui, partant de l'individu et de ses désirs, explique l'émergence de ce phénomène alors que la théorie walrassienne vise à déterminer directement le niveau des prix d'équilibre d'une situation particulière.

Focus mengerien sur le processus d'émergence du phénomène versus focus walrassien sur la description du phénomène en situation d'équilibre. Bien évidemment, les systèmes d'équations simultanées sont d'un usage limité eu égard à la première question, mais sont en revanche totalement adaptés à la résolution de la seconde préoccupation. Il semble donc plus opportun de caractériser le rejet de Menger pour les mathématiques non comme une position de principe mais comme une position plus articulée, dans la mesure où les outils formels alors disponibles n'étaient pas adaptés à sa conception de l'explication économique.

11. Menger donne l'exemple suivant : supposons que l'agent A dispose de 100 unités de bien A, qu'il serait prêt à les échanger contre non moins de 40 unités de bien $\mathrm{B}$ et qu'il trouve un agent $\mathrm{B}$, prêt à échanger les 40 unités de bien $\mathrm{B}$ à sa disposition contre non moins de 80 unités de bien $\mathrm{A}$, alors le prix relatif du bien B en termes de bien A se situera entre 80 et 100 (Menger [1871], 1950, p. 194). 
Dire de Menger qu'il représente une simple variante littéraire de Walras n'est pas simplement réducteur mais faux.

Cette question du rejet des mathématiques se révèle fondamentale. Ingrao et Israel ([1990], p. 36) nous rappellent que l'ambition de Walras est d'appliquer la démarche scientifique de Newton à l'économie. En accord avec la culture post-Renaissance française qui situe la philosophie scientifique de Newton au centre des progrès de la société toute entière, le leader marginaliste considère les mathématiques comme quelque chose de bien plus important qu'un simple outil démonstratif lui permettant de donner une représentation plus simple et rigoureuse d'un équivalent littéraire. Les mathématiques sont pour lui en effet un outil d'investigation, une véritable " méthode de recherche » : " Il est naturel à qui n'est pas mathématicien, de croire que la forme mathématique, là où elle peut être employée, ne donne rien de plus que le langage ordinaire, et sert seulement à faire comprendre les choses à ceux qui ne les saisiraient que sous cette forme ; mais cette assertion fera sourire tout homme au courant des services rendus par les mathématiques à toutes les sciences auxquelles on a pu les appliquer : mécanique, astronomie, physique, chimie » (lettre de Walras du 16 janvier 1882, citée dans Antonelli [1953], p. 269, n. 3).

C'est à ce niveau précis que se situe le clivage avec Menger : «L'objet de mes recherches est de ramener les phénomènes compliqués de l'économie à leurs véritables causes, et la recherche des lois d'après lesquelles lesdits phénomènes compliqués de l'économie politique se reproduisent. Les résultats de mes recherches peuvent être revêtus de formules mathématiques. Les représentations mathématiques peuvent contribuer à leur démonstration : cependant la méthode mathématique de représentation n'appartient en aucune matière à l'essentiel de la tâche que je me suis proposée » (lettre de Menger du $1^{\text {er }}$ juin 1883, citée dans Antonelli [1953], p. 272).

Comme le souligne également Garrouste [1994], la méthode mathématique de Walras n'est simplement pas adaptée à l'objectif de Menger qui est de déterminer l'essence des phénomènes économiques compliqués. Dès lors que l'ambition du théoricien est de comprendre le processus d'émergence d'un phénomène à travers une décomposition causale en termes de ses éléments les plus simples, la formalisation sous forme d'équations simultanées est inappropriée dans la mesure où elle ignore purement et simplement la séquence portant au phénomène, en se concentrant exclusivement sur les propriétés du résultat final du processus, l'équilibre. Comprendre un phénomène revient pour Menger à être capable de reproduire le processus qui mène à son existence, à le construire. Comprendre un phénomène pour Walras revient à montrer que son existence est logiquement compatible avec son environnement.

\section{SPÉCIFICITÉ ONTOLOGIQUE ET POSSIBILITÉ D'UN PROGRAMME NÉOMENGERIEN}

L'ontologie sociale est l'étude de l'essence de la réalité sociale - pour nous de la réalité économique. La confrontation menée au cours de cet article permet de caractériser Menger et Walras autour de deux visions distinctes de la réalité économique qu'ils analysent. 
La réalité formalisée par Walras et les marginalistes est celle d'un monde où les configurations économiques sont le résultat de l'interaction d'automates aux conduites prédéterminées dans un environnement sans facteur de perturbation, c'està-dire un monde fermé d'agents autonomes prévisibles en interaction. À l'opposé, Menger et, par la suite, les économistes autrichiens qui poursuivent son programme dans la lignée du subjectivisme radical insistent sur la dimension créatrice des acteurs économiques, non réductibles à de simples réacteurs : les agents mengeriens sont engagés dans un processus d'acquisition de connaissances (connaissances relatives à la façon de produire les biens, à la nature des besoins des autres agents, à la nature des biens susceptibles de satisfaire ces besoins, etc.) qui les amène à continuellement modifier leurs plans d'actions ; les agents mengeriens vivent dans un monde d'incertitude où ils peuvent faire des erreurs; les décisions sont basées sur les anticipations que les agents forment à propos d'un futur inconnu et à travers lesquelles s'expriment leurs capacités créatrices. La réalité économique autrichienne est appréhendée comme un système ouvert caractérisé par des changements inattendus.

Le rejet autrichien de la formalisation prend ainsi une nouvelle dimension et perd définitivement son caractère anecdotique. Il résulte d'une adhésion sans concession à une conception de la réalité sociale difficilement formalisable en termes de fonctions dérivables et continues. Ces outils mathématiques ne sont pas compatibles avec les soubassements ontologiques de l'analyse mengerienne et de l'approche causale-génétique qui en découle. Au contraire, ces outils sont tout à fait adaptés à l'approche fonctionnelle, toujours pour reprendre les termes de Mayer, à laquelle demeureront fidèles les disciples de Walras. Il est naturel que des théories fondées à partir de deux points de départ si dissemblables soient irrémédiablement éloignées. Cela ne signifie pas que la vision mengerienne ne puisse pas être formalisée. Il existe au sein des mathématiques des courants philosophiques associés à des positions ontologiques spécifiques et distinctes. En particulier, il est possible de distinguer deux familles d'outils mathématiques, les outils formalistes et les outils constructivistes, les premiers étant associés à l'ontologie sociale liée au déductivisme alors que les seconds seraient adaptés à la vision autrichienne de systèmes ouverts dynamiques. Ainsi, si la position de Menger à l'égard des mathématiques semblait justifiée par le fait que les outils alors disponibles n'étaient pas adaptés, la position des auteurs modernes risque à juste titre d'être taxée de dogmatique si aucune tentative n'est faite pour modéliser leurs approches avec des outils spécifiques et compatibles avec l'optique causale-génétique. Il est difficile en particulier de ne pas être frappé par la proximité entre la démarche autrichienne et la démarche multi-agent telle que la présente l'un de ses plus fameux exposants, Epstein ([2006], p. 1587) $)^{12}$ : «[...] pour expliquer un macrophénomène, il est nécessaire de montrer comment il pourrait émerger au sein d'une société réaliste. Il faut démontrer comment un ensemble d'agents identifiables, hétérogènes, autonomes, à la rationalité limitée et interagissant localement, pourraient y parvenir dans un délai raisonnable. La modélisation par simulation multi-agent est un instrument spécialement puissant pour construire de telles démonstrations génératives suffisantes. »

Les sciences de la complexité font partie des sciences dites génératives, par opposition au positivisme traditionnel auquel demeure rattachée la science économique néoclassique. Une science générative prétend comprendre un phénomène à

12. Vriend [2002], Nell [2010] et Seagren [2011] montrent la compatibilité entre l'approche autrichienne et l'outil du multi-agent. 
travers sa construction, son élaboration, alors que les positivistes prêtent attention à la consistance logique de la modélisation formelle qu'ils proposent du phénomène. Ainsi, dans le cadre de la complexité, comprendre le processus de marché signifie être capable de le reproduire, par simulation ou à travers l'identification de l'algorithme qui mène à son émergence, en partant des entités autonomes dotées de règles d'interaction simples et plausibles dans un environnement spécifique. Pour l'approche positiviste dominante, comprendre le marché signifie démontrer que l'équilibre concurrentiel existe, ou plutôt que sa non-existence mènerait à une contradiction ; dans ce cadre, l'équilibre est une nécessité logique et représente la finalité de l'analyse indépendamment de la façon dont cet équilibre est éventuellement atteint. L'approche générative fait quant à elle écho à la démarche causale-génétique empruntée par Menger et dont les Autrichiens modernes ont hérité. Le fossé qui sépare Walras de Menger n'est ainsi pas réductible à une simple différence de forme.

C'est la reconnaissance des éléments de dissension entre Menger et Walras qui permet aujourd'hui de contribuer, grâce à la disponibilité d'outils désormais appropriés, au développement d'une nouvelle théorie microéconomique. Si donc, comme argumenté tout au long de cet article, la perspective mengerienne est bel et bien fondée sur une représentation totalement distincte de la réalité économique de celle de Walras, avec pour conséquence d'engendrer deux programmes de recherche distincts et basés sur l'usage de méthodologies et d'outils distincts, il est temps de répondre maintenant à la question de la modernité de Menger et de décrire les principales lignes de développement d'un programme néomengerien.

Tout d'abord, quelle est la question centrale de ce programme ? Comme indiqué précédemment, ce n'est que dans son ouvrage de 1883 que Menger indique que l'objet central de la théorie économique est la compréhension théorique de l'origine et du changement des institutions organiquement créées. Le marché est l'institution organique économique par excellence et comprendre le processus de marché est au cœur de l'agenda néomengerien ici défini ${ }^{13}$.

Comprendre un phénomène dans la perspective générative néomengerienne consiste à être capable de le reproduire de façon plausible en partant des éléments constitutifs les plus simples, à savoir les agents économiques dotés d'une rationalité définie autour du principe de l'accomplissement dans un environnement en continuelle mutation où les informations sont dispersées. L'objectif est donc de simuler le processus d'interaction économique en identifiant des règles de comportement réalistes sans intervention de mécanisme de coordination centralisé.

Le programme néomengerien aborde sans ambiguïté l'économie comme science complexe et la simulation multi-agent permet, pour reprendre les termes de Tesfatsion [2006], une étude quantitative des aspects mis en avant par la tradition autrichienne d'un point de vue exclusivement qualitatif ${ }^{14}$. Il ne s'agit

13. Cette tentative de définition se distingue de Wagner [2010] qui, bien que partant également des intuitions mengeriennes génératives, réduit la portée de ce programme à l'apport de la nouvelle économie politique. Nous proposons de mettre au cœur de ce programme la question de l'analyse du processus de marché.

14. La proximité de l'école autrichienne avec l'économie de la complexité n'est pas un thème nouveau. Mais il s'agit le plus souvent de trouver chez Hayek quasi exclusivement les prémisses d'une théorie économique de la complexité (Caldwell [2004]; Koppl [2006], [2009] ; Lewis [2012]) ou alors, à l'opposé, de considérations très générales faisant de la complexité le trait central de la tradition autrichienne ; Rosser [2010] refuse cette généralisation et distingue de façon plus fine parmi les auteurs autrichiens ceux qui développent une approche comportant des prémisses de complexité (Menger et Hayek) de ceux chez qui ces considérations sont totalement absentes (Böhm-Bawerk). 
pas ici de fournir une théorie du processus de marché. L'ambition du théoricien néomengerien est redimensionnée : il s'agit plutôt d'offrir différentes théories des processus de marché empiriquement ancrées dans une typologie des différents mécanismes concurrentiels.

À chaque type de marché correspond un modèle spécifique basé sur une taxonomie d'agents économiques, chacun caractérisé 1) par un ensemble de données de départ, accessibles ou non, en totalité ou en partie, aux autres agents ou groupe d'agents et 2) par des méthodes comportementales pouvant être représentées par un ensemble d'algorithmes spécifiques ; chaque type de marché est également basé sur l'identification de l'environnement institutionnel au sein duquel ces agents interagissent. L'environnement institutionnel est composé de plusieurs strates. Il est nécessaire d'identifier pour chaque type idéal de marché les institutions externes et les institutions internes qui le caractérisent. Il s'agit d'une distinction posée par Lachmann ([1970], p. 90), le plus mengerien des autrichiens modernes, et qui prend en compte le caractère plus ou moins flexible des institutions au sein desquelles les individus interagissent : les institutions externes fournissent un cadre de référence stable et rigide qui permet aux individus de prendre leurs décisions sans avoir à connaître a priori tous les détails des plans d'action des autres acteurs ; les institutions internes sont quant à elles flexibles et coévoluent en réponse à l'interaction individuelle.

Au-delà des différents types idéaux de marchés, la logique globale est identique : le marché est analysé comme un processus continu qui résulte de l'interaction d'agents à la rationalité limitée évoluant dans un environnement incertain en constante évolution. Les individus se basent pour prendre leurs décisions sur l'élaboration de plans fins-moyens (principe de l'accomplissement) ; ces plans sont formés à partir des informations locales dont ils disposent, des connaissances qu'ils élaborent sur la base de leurs expériences et observations, et des anticipations qu'ils imaginent à propos du futur. Les institutions externes servent de points de repère venant limiter l'incertitude radicale au sein de laquelle les individus interagissent et les institutions internes sont elles-mêmes affectées par les interactions individuelles. Lorsque la confrontation des plans individuels laisse apparaître des problèmes de coordination, des erreurs, des plans non réalisés, ceux-ci sont révisés sur la base des nouvelles informations et connaissances disponibles selon des règles comportementales elles-mêmes adaptées de l'expérience passée.

Le modèle doit être élaboré de sorte à être dynamiquement complet dans le sens où le modélisateur n'a plus à intervenir une fois la simulation lancée. La « résolution " d'un modèle prend une forme différente de l'acceptation traditionnelle puisque l'aboutissement de la simulation n'est pas (ou très rarement) une situation d'équilibre, mais l'émergence de quelques régularités ou schémas stables rendant le processus de marché viable. Il est important à ce niveau de noter que ce programme néomengerien ne s'appuie pas sur la catégorie hayekienne d'ordre spontané et lui préfère celle d'émergence qui ne véhicule pas de valeurs implicites d'efficience ${ }^{15}$. Les propriétés émergentes peuvent avoir des implications positives ou négatives pour les agents concernés et la supériorité de la décentralisation sur la régulation n'est pas préétablie mais objet d'investigation. La question de l'efficience est ainsi abordée non plus en relation avec les standards paretiens mais à travers l'analyse des propriétés du réseau d'interaction (réseau régulier, small world ou random) qui émerge de la simulation.

15. Sur cette distinction entre émergence et ordre spontané, voir en particulier Axtell [2007]. 


\section{CONCLUSION}

Vingt ans après la publication de l'article de Jaffé [1976], le débat a été réalimenté par diverses tentatives de réhomogénisation : Peart [1998] montre la proximité de l'analyse du consommateur de Menger avec celle de Jevons, et Hébert [1998] se place à un niveau de généralité tel qu'il réunifie les trois protagonistes autour de leur volonté commune de rompre avec le programme classique macroéconomique. Cet article poursuit au contraire l'ambition de Jaffé et vise à vérifier sa proposition selon laquelle le passage du temps révélera que ce qui différencie Menger de Walras est bien plus important que ce qu'ils ont en commun. Le temps est passé et si la plupart des potentialités du programme walrassien ont été discutées, critiquées et exploitées grâce en particulier à la disponibilité d'outils mathématiques adaptés, les potentialités du programme mengerien sont encore en grande partie à explorer. La structure de connaissances élaborée par Menger ne pouvait être exploitée à partir des outils mathématiques fonctionnels alors disponibles, mais les outils de la complexité et notamment la modélisation multi-agent permettent aux économistes de renouer avec le programme mengerien pour contribuer au développement de la microéconomie moderne en termes computationnels.

Notre ambition n'a pas été de faire une relecture a posteriori de Menger afin d'en faire un précurseur de l'économie comme un système complexe, mais plutôt de le présenter comme source d'inspiration potentielle pour la microéconomie moderne grâce à la disponibilité de nouveaux outils adaptés à sa conception de la réalité économique qui peuvent venir renouveler et approfondir ses théories.

\section{RÉFÉRENCES BIBLIOGRAPHIQUES}

Álvarez A. et Bignon V. [2013], « L. Walras and C. Menger: Two Ways on the Path of Modern Monetary Theory », The European Journal of the History of Economic Thought, 20 (1), p. 89-124.

Antonelli E. [1953], « Le souvenir de Léon Walras : Léon Walras et Carl Menger à travers leur correspondance », Économie appliquée, 6, p. 269-287.

Arena R. et Gloria-Palermo S. [2008], « Walras and Menger on Money: A Comparative View », History of Political Economy, 40 (2), p. 317-343.

ARENA R. et RAGNi L. [1994], "Libre concurrence et méthodologie walrassienne : une tentative de mise en relation », Économies et Sociétés, 20-21 (10-11), p. 161-182.

Axtell R. L. [2007], «What Economic Agents Do: How Cognition and Interaction Lead to Emergence and Complexity », The Review of Austrian Economics, 20 (2-3), p. 105-122.

Bridel P. et Mornati F. [2009], " De l'équilibre général comme "branche de la métaphysique". Ou de l'opinion de Pareto sur le projet walrassien ", Revue économique, 60 (4), p. 869-890.

CAldwell B. [2004], « Some Reflections on F. A. Hayek's The Sensory Order », Journal of Bioeconomics, 6 (3), p. 239-254.

Epstein J. M. [2006], « Remarks on the Foundations of Agent-Based Generative Social Science ", dans L. Tesfatsion et K. L. JudD (dir), Handbook of Computational Economics, Amsterdam, Elsevier, p. 1585-1604.

Fontaine P. [1998], « Menger, Jevons, and Walras Un-Homogenized, De-Homogenized, and Homogenized: A Comment on Peart », The American Journal of Economics and Sociology, 57 (3), p. 333-340. 
Gloria-Palermo S. [1999], The Evolution of Austrian Economics: From Menger to Lachmann, Londres, Routledge.

Garrouste P. [1994], « Carl Menger et Léon Walras à propos de l'utilisation des mathématiques en économie politique », Économies et Sociétés, 28 (10), p. 11-27.

Hébert R. F. [1998], « Jevons and Menger Re-Homogenized: Who Is the Real "Odd Man Out"? », The American Journal of Economics and Sociology, 57 (3), p. 327-332.

Ingrao B. et Israel G. [1990], The Invisible Hand: Economic Equilibrium in the History of Science, Cambridge (Mass.), The MIT Press.

JACOUD G. [1994], « Stabilité monétaire et régulation étatique dans l'analyse de Léon Walras », Revue économique, 45 (2), p. 257-288.

JAFFÉ W. (DIR.) [1965], Correspondence of Léon Walras and Related Papers, Amsterdam, North Holland, vol. I-III.

JAFFÉ W. [1976], « Menger, Jevons and Walras De-Homogenized », Economic Inquiry, $14(4), 511-524$.

Koppl R. [2006], «Austrian Economics at the Cutting Edge », Review of Austrian Economics, 19 (4), 231-241.

Koppl R. [2009], « Complexity and Austrian Economics », dans J. B. Rosser (dir), Handbook of Complexity Research, Cheltenham, Edward Elgar, p. 393-408.

Lachmann L. [1970], The Legacy of Max Weber, Londres, Heinemann.

Lachmann L. [1976], « From Mises to Shackle: An Essay on Austrian Economics and the Kaleidic Society », Journal of Economic Literature, 14 (1), 54-32.

Latzer M. et Schmitz S. (DIR.) [2002], Carl Menger and the Evolution of Payments Systems, Cheltenham, Edward Elgar.

LEwIs P. [2012], « Emergent Properties in the Work of Friedrich Hayek », Journal of Economic Behavior \& Organization, 82 (2-3), p. 368-378.

Mayer H. [1932], « Der Erkenntniswert der Funktionellen Priestheorien ». Trad. angl. : "The Cognitive Value of Functional Theories of Price: Critical and Positive Investigations Concerning the Price Problem », dans I. M. KIRZNER (dir.), Classics in Austrian Economics: A Sampling in the History of a Tradition, Londres, Pickering and Chatto, 1994, vol. II, p. 249-280.

MÉnARD C. L. [1979], «Équilibre, déséquilibre, temps : un peu d'histoire », Économie appliquée, 32 (2-3), p. 229-251.

Menger C. [1871], Grundsätze der Volkswirthschaftslehre, Vienne, Wilhelm Braumüller. Trad. angl. : Principles of Economics, Glencoe, The Free Press, 1950.

Menger C. [1883], Untersuchungen über die Methode der Socialwissenschaften und der Politischen Oekonomie insbesondere, Leipzig, Dunker \& Humblot. Trad. angl. : Problems of Economics and Sociology, Urbana, University of Illinois Press, 1963 ; trad. fr. : Recherches sur la méthode dans les sciences sociales et en économie politique en particulier, Paris, Éditions de l'EHESS, 2011.

Menger K. [1973], « Austrian Marginalism and Mathematical Economics », dans J. HicKs et W. Weber (dir.), Carl Menger and the Austrian School of Economics, Oxford, Clarendon Press, p. 38-60.

Merton R. K. [1973], The Sociology of Science, Chicago, The University of Chicago Press.

Moscati I. [2013], « Were Jevons, Menger, and Walras Really Cardinalists? On the Notion of Measurement in Utility Theory, Psychology, Mathematics, and Other Disciplines, 1870-1910 », History of Political Economy, 45 (3), p. 373-414.

Nell G. L. [2010], « Competition as a Market Process: An Austrian Rationale for AgentBased Modeling », The Review of Austrian Economics, 23 (2), p. 127-145.

O’Driscoll G. et Rizzo M. [1985], The Economics of Time and Ignorance, Oxford, Blackwell.

PeART S. [1998], « Jevons and Menger Re-Homogenized? Jaffé Twenty Years After », The American Journal of Economics and Sociology, 57 (3), p. 307-325.

Rebeyrol A. [1999], La pensée économique de Walras, Paris, Dunod.

Rizzo M. J. [1994], « Time in Economics », dans P. BoetTKe (dir.), The Elgar Companion to Austrian Economics, Cheltenham, Edward Elgar, p. 111-117. 
Rosser J. B. [2010], « How Complex Are the Austrians ? », dans R. Koppl, S. Horwitz et P. Desrochers (dir.), What Is so Austrian about Austrian Economics?, Bingley, Emerald Group, p. 165-179.

Schumpeter J. A. [1954], History of Economic Analysis, Londres, George Allen and Unwin. Trad. fr. : Histoire de l'analyse économique, Paris, Gallimard, 1983, 3 t.

Seagren C. W. [2011], « Examining Social Processes with Agent-based Models », The Review of Austrian Economics, 24 (1), p. 1-17.

Streissler E. [1972], « To What Extent Was the Austrian School Marginalist? », History of Political Economy, 4 (2), p. 426-441.

Tesfatsion L. [2006], «Agent-Based Computational Economics: A Constructivist Approach to Economic Theory », dans L. Tesfatsion et K. L. JudD (dir.), Handbook of Computational Economics, Amsterdam, Elsevier, vol. II, p. 831-880.

VRIEnd N. J. [2002], « Was Hayek an ACE ? », Southern Economic Journal, 68 (4), p. 811-840.

Wagner R. E. [2010], Mind, Society, and Human Action: Time and Knowledge in a Theory of Social Economy, Londres, Routledge.

WALKER D. A. [1987], « Walras's Theories of Tatonnement », Journal of Political Economy, 95 (2), p. 758-774.

Walras L. [1874a], Eléments d'économie politique pure, ou Théorie de la richesse sociale,

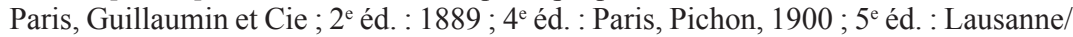
Paris, Rouge / Pichon et Durand-Auzias, 1926 ; rééd. de la 4éd : Paris, Librairie générale de droit et de jurisprudence, 1952 ; rééd. : vol. VIII de A. WALras et L. WALRAS, Euvres économiques complètes, Paris, Economica, 1988.

Walras L. [1874b], « Principe d'une théorie mathématique de l'échange », Journal des économistes, 34 (100), 15 avril, p. 5-22, rééd. dans L. Walras, Mélanges d'économie politique et sociale, vol. VII de A. WALras et L. WaLras, Euvres économiques complètes, Paris, Economica, 1987.

Walras L. [1886], Théorie de la monnaie, Lausanne, Corbaz et Cie, rééd. : vol. X de A. Walras et L. Walras, Euvres économiques complètes, Paris, Economica, 1992.

Walras L. [2000], Euvres diverses, vol. XIII de A. Walras et L. Walras, Euvres économiques complètes, Paris, Economica. 